\title{
SOBRE LA OPORTUNIDAD DE LAS TASAS TURÍSTICAS: EL CASO DE SEVILLA
}

\author{
Ana M. García López \\ Manuel J. Marchena Gómez. \\ Álvaro Morilla Maestre \\ Universidad de Sevilla
}

\section{RESUMEN}

Este artículo recoge la eclosión del debate sobre las tasas turísticas en España y Europa, con especial énfasis en Sevilla para su aplicación. La fiscalidad propia sobre el turismo es más amplia de lo que aparenta, y se correlaciona con un afán recaudatorio en lugares de mayor colmatación turística, con problemas de impacto ambiental por sobredosis de oferta, y en general, para mejorar la caja de marketing. Desde luego, el instrumento fiscal más utilizado es la tasa hotelera de pernoctación. Sin embargo, en los últimos años se ha derivado la visión finalista de la tasa turística hacia derroteros paradójicamente no propiamente turísticos. Más bien se pretende con la recaudación de esta tasa a turistas resolver el agotamiento de la vía pública, intervenciones en el paisaje urbano para optimizar el destino o el propio de los residentes, que sufren el impacto turístico. Se trata el debate abierto y aún sin cristalizar en Sevilla. El ayuntamiento ha iniciado la propuesta sobre la tasa, y la Comunidad Autónoma que es la competente, lo ha rechazado. En el discurrir de los hechos se observa que son los hoteleros los máximos oponentes a la tasa, en un contexto que no debe olvidarse de máxima difusión de apartamentos turísticos alegales e ilegales. Se analiza la posición de distintos actores, así como los datos 2008-2015 y los logros y retos a partir de 2016: sin discusión, el año histórico del turismo en Sevilla.

Palabras clave: Fiscalidad turística; tasas turísticas; tasa de pernoctación hotelera; destino turístico urbano; sostenibilidad; Sevilla.

Fecha de recepción: 29 de mayo de 2017.

Fecha de aceptación: 22 de enero de 2018.

Facultad de Turismo y Finanzas. Universidad de Sevilla. Avda. San Francisco Javier, s/n. 41018 SEVILLA (España).E-mail: angar@us.es,mmarchena@us.es, alvmormae@us.es 


\title{
About the opportunity of the tourist taxes: the case of Seville
}

\begin{abstract}
The aim of this paper is to present the different viewpoints on the existing debate over tourism taxes in Spain and Europe; with a special focus on Seville, for its application. Tourism taxation itself is broader than it seems and it's correlated with an effort to raise income for extra marketing promotion; especially in saturated tourism destinations, which usually suffer from environmental problems due to excessive offer. Surely, the most used tourism tax tool is the hotel overnight rate; however, paradoxically, in recent years, this income has been used in matters different than tourism. The application of this overnight stay tax for tourists (it would be unlikely for visitors or day excursionist pay this tax), has been the improvement of public roads, water facilities, and urban landscape to optimize the destination Tourist or the residents, who undergo the tourist impact in excess. It is, in detail, the open debate and still without crystallizing, on the case of Seville. The council has initiated the proposal on the rate, and the Autonomous Community that is the competent, has rejected it. In the course of events it is observed that hoteliers are the highest opponents of the rate; In a context that should not be forgotten about the extraordinary diffusion of the tourist apartments, both alegals and properly illegal. It will be seen in the text through qualified interviews, the position of the different actors, and we provid the data for 20082015, and the successes and challenges from 2016: the historic year of tourism in Seville.
\end{abstract}

Keywords: Tourism Taxation; tourist taxes; hotel overnight tax (room tax); urban tourist destination; sustainability; Seville.

\section{INTRODUCCIÓN: ASPECTOS DE CONTEXTO}

El debate sobre las tasas en turismo, particularmente la más utilizada, la tasa de pernoctación hotelera debe enmarcarse en la actualidad, no sólo en el hecho recaudatorio, sino en una pléyade de factores que cómo casi todo en el turismo mirado como destino, es de carácter transversal. ¿Es realmente la tasa turística una herramienta útil para la sostenibilidad? ¿Qué sostenibilidad: ambiental sólo, o también la económica, o sólo como aportación a la promoción del lugar?

Tres hechos deben contextualizar la reflexión sobre bases empíricas (nos ocuparemos del caso de Sevilla) para concluir sobre la oportunidad de la fiscalidad turística. Mejor cuatro, porque el primero es general y parte de la crisis del euro en 2008 y la depreciación de las políticas fiscales progresivas en general, para en todo caso agrandar los impuestos y tasas regresivas como el Impuesto del Valor Añadido. Las Tasas turísticas se encuentran en este sitio: el turista paga igual sin contarse con su capacidad adquisitiva. Sólo se discrimina en algunos lugares por las estrellas hoteleras.

En segundo lugar, el contexto actual procíclico en turismo, ha abundado en destinos maduros sobre una evidencia empírica en la saturación. La fiscalidad turística vendría a paliar el discomfort local con respecto al turismo. Tratando de penalizar dicha saturación y ejecutando acciones de mejoras en el impacto turístico para los residentes. Amén del mercadeo más selectivo del destino. Paradigmático es Amsterdam. 
En tercer lugar, no debe olvidarse la explosión del alojamiento turístico no regulado o regulado a duras penas en forma de vivienda, y la percepción del mundo hotelero en todas sus gamas, de discriminación negativa en presión fiscal, ordenación e inspección turística. Sobre todo en un proceso de salida de la crisis, y mayor rol de los prescriptores de intermediación digital y el low cost.

Por fin, en cuarto lugar, pocos sectores económicos son beneficiados por la promoción pública como el turismo a través de los fondos para el mercadeo del destino turístico, sin embargo, el sector turístico privado, es muy renuente en colaborar o retornar ingresos por dichas políticas. $\mathrm{O}$ menos aún, en colaborar activa y financieramente en actividades o eventos (alargar la Feria de Abril de Sevilla en fines de semana, por ejemplo), o en obras públicas de claro componente turístico. En las siguientes páginas abordaremos la oportunidad de la tasa turística, poniendo como ejemplo Sevilla; con énfasis en ubicar dicho debate en el contexto más actual de territorios turísticos en proceso de desbordar su capacidad de carga. Como se ha aludido, empiezan a aparecer signos alarmantes en destinos urbanos, de disfuncionalidades e insostenibilidades turísticas, señal inequívoca de problemas futuros. Cada vez parece más evidente que se está produciendo una burbuja tanto hotelera, basada en el interés de los Fondos de Inversión más agresivos, como residencial, con la proliferación en barrios muy concretos de viviendas turísticas de muy alta rentabilidad y escasa aportación fiscal. No debe olvidarse que el sector público proporciona seguridad, transportes y servicios a esos turistas, amén de paisaje urbano, a cambio en la mayoría de los casos de una tasa o IVA, para los apartamentos, con el tipo más bajo que existe: cero.

\subsection{Las Tasas turísticas y su expansión}

Desde que a principios del siglo XX Francia estableciera la primera taxe de séjour, los tributos al turismo han proliferado, sobre todo en las dos últimas décadas bajo diferentes formas. Numerosa literatura recoge el interés por un tema de estudio tan controvertido como mediático (Abeyratne, 1993; Bird, 1992; Bokobo, 1999; ETOA, 2016; Fish, 1982; Font, 2012; Franco, 1996; Gooroochurn \& Thea, 2005; Hall, 2008; Gago et al, 2009; Jensen \& Wanhill, 2002; Piga, 2003; Shen \& Tsui, 2009; OMT, 1998). Tanto desde la economía y el derecho, como desde la geografía y el turismo.

El número de países que gravan la actividad turística internacional se incrementa cada año, a través de distintas fórmulas, denominaciones (tasas, impuestos o contribuciones) y cuantías. Si bien podemos establecer con carácter general los tres modelos más implementados en la actualidad en los destinos turísticos, con independencia de que sean urbanos o de litoral. Las tasas por pernoctación, gravan directamente al turista alojado en establecimientos hoteleros. Es la más extendida en Europa, especialmente en destinos urbanos consolidados. Se cobra en función del número de noches (hasta un máximo de 7-10 noches dependiendo del destino) y de la categoría del alojamiento, habiendo excepciones para los menores de 12-16 años, estudiantes de idiomas menores de 18 años, turistas nacionales de negocios, etc. Las tasas aéreas, por su lado, gravan mediante un impuesto en el aeropuerto la entrada o la salida del país sólo si se utiliza este medio de transporte. Alemania, EEUU, Tailandia, Camboya o Marruecos entre otros la aplican desde hace años en sus territorios. Y las tasas de entrada/salida. Frecuentes en destinos americanos tales como 
México, Costa Rica, Cuba, República Dominicana o Venezuela, entre otros. Más elevadas cuanto más demandado el destino, predominando los caribeños del sol y playa (Punta Cana o Riviera Maya). Otros destinos, utilizan una diferente denominación para gravar la misma salida del turista. Es el caso de Túnez, que obliga a adquirir al turista un "sello de solidaridad" (Canalis, 2014).

Hay destinos donde el gravamen es doble, tal es el caso de los Estados Unidos (Fuji et al, 1985; Mak \& Nishimura, 1979; Mak, 1988), que además de una tasa de entrada, cobra por pernoctación hotelera según Estado y ciudad, oscilando entre el 5-7,25\% sobre la tarifa de la habitación. O más recientemente Malta, que junto a los 3,5 euros/entrada, ha implementado en alojamientos la eco-tax (0,50 euros/noche).

Los destinos emergentes asiáticos, de Oriente Medio y Australia también han trabajado en esta misma línea impositiva (Gooroochurn \& Sinclair, 2005; Ihalanayake, 2007; Ponjan \& Thirawa, 2016), aunque no siempre con éxito. Frente a ellos, otros tradicionales como Egipto y Malasia, o más recientemente las islas Maldivas y Dubái sí lo han consolidado (Hosteltur, 2014).

A una desigual distribución espacial hay que sumar una heterogénea finalidad en lo que a importes recaudados se refiere: mientras que una parte de los países lo dedican al mantenimiento y conservación del patrimonio monumental o a la recuperación del daño ecológico derivado de la presión antrópica, la mayor parte ellos lo invierten en promoción turística.

De todas las formas de gravar la actividad turística actualmente en vigor, sin duda la que más ha proliferado ha sido la primera, de ahí que existan precedentes de investigaciones a este respecto desde los años 70 y 80 del pasado siglo (Bonham et al, 1991; Crotss \& McGrill, 1994; Hiemstra \& Ismail, 1992; Hiemstra \& Ismail, 2001; Mak \& Nishimura, 1979; Mak, 1988; Spenger \& Uysal, 1989). Y es el continente europeo quien más la ha adaptado en sus destinos. Siendo la región que mayor volumen de turistas internacionales registra anualmente, más de 600 millones en 2016, constituye históricamente el principal destino turístico receptor del mundo (OMT, 2017). Y sus ciudades y reposicionamiento competitivo vienen siendo objeto de numerosas investigaciones (Asshworth \& Page, 2011). En la figura 1 puede observarse los países donde existen tasas e impuestos en alojamientos turísticos, ya implantadas en las capitales y destinos urbanos más internacionales, con la excepción de Londres.

Junto a destinos urbanos de Francia, Croacia, Italia, Alemania, España, Bélgica, Holanda y Portugal, otras ciudades europeas de Austria, Hungría, Suiza, Bulgaria, Eslovenia, Lituania, Polonia, Rumanía y la República Checa están aplicando más recientemente estas tasas de pernoctación hotelera. A modo de ejemplo, para una familia de cuatro miembros durante una estancia de siete días (6 noches) en alojamiento de categoría 4 o $5^{*}$, el incremento económico de la misma oscilaría desde los 28 o 30,48€ (de Dubrovnik y Liubljiana, respectivamente) hasta los $196 €$ (en Roma o Bruselas).

Por otro lado, países donde no hay establecido este tributo son Finlandia, Estonia, Grecia, Irlanda, Letonia, Luxemburgo, Noruega, Suecia, Reino Unido y Dinamarca. Si bien se han realizado estudios de impactos e investigaciones aplicadas en algunos de ellos (Durbarry \& Sinclair, 2001; ETOA, 2015; HUGHES, 1981; Jensen \& WANHILL, 2002;). 


\section{Figura 1 \\ PAÍSES EUROPEOS CON TASAS DE ALOJAMIENTOS EN DESTINOS URBANOS}

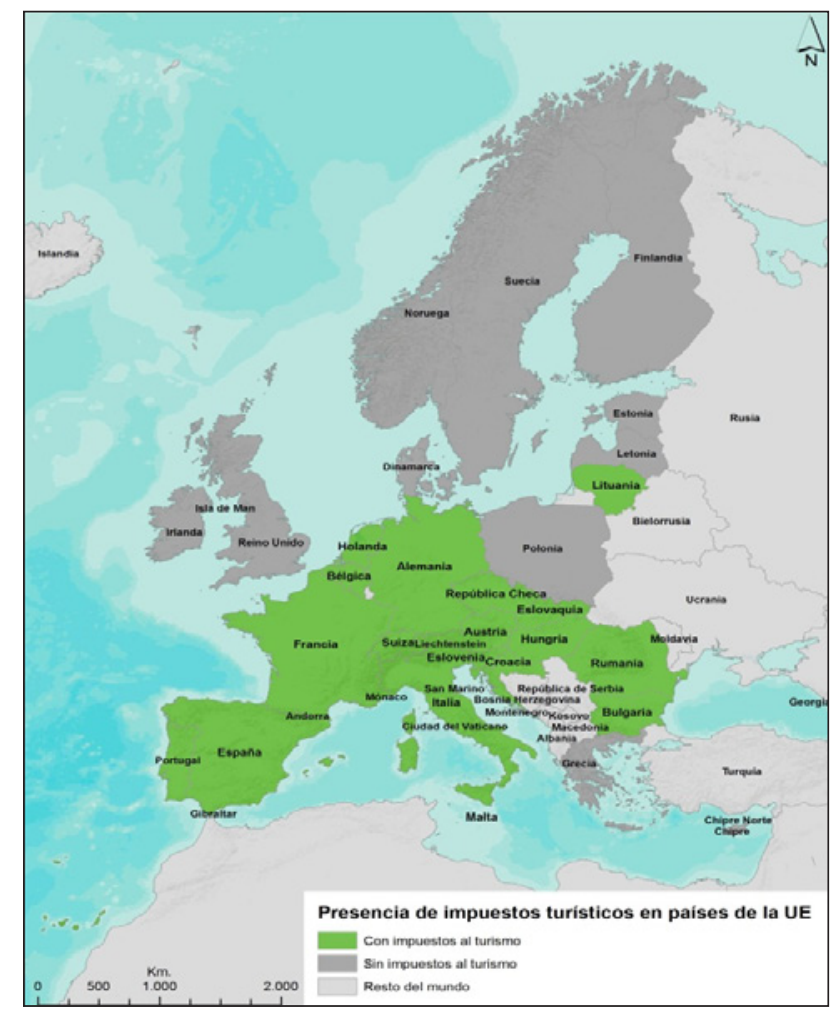

Fuente: elaboración propia. Datos ETOA, 2016.

La tabla 1 muestra destinos turísticos urbanos europeos y las tarifas referidas a sus tasas por pernoctación:

Las cuantías por esta contribución varía no sólo dependiendo de la categoría de los alojamientos, sino y sobre todo, en función de la importancia y peso turístico de los propios destinos. Medido esto último en términos de volumen de demanda. Sólo la capital británica queda al margen de esta realidad. Si bien es cierto que se ha planteado muy recientemente con la oposición en bloque del sector hotelero (2100 hoteles); y es que la ciudad más turística de Europa ya cobra el IVA más alto del continente en sus hoteles, por lo que un impuesto extra (5\%) está siendo muy cuestionado (Daley, 2017).

Para países donde ya existen estas tasas desde hace años, la falta de definición o criterios comúnmente aceptados dentro de un mismo territorio nacional, comienza a generar polémica, especialmente en relación al destino de los fondos. Mientras que para los destinos que actualmente se lo plantean como posibilidad, el principal debate gira en torno a la posible pérdida de competitividad turística en términos de demanda. 
Tabla $\mathbf{n}^{0} 1$

TASAS POR PERNOCTACIÓN EN DESTINOS

\begin{tabular}{|c|c|c|}
\hline Destino & Denominación & Var. Impuesto (aprox.) \\
\hline Amberes & City Tax & $2 €$ \\
\hline Ámsterdam & Toeristembelasting & $5 \%$ precio por pernoct. \\
\hline Barcelona & Tasa turística/ IEET* & $0,75-2,50 €$ \\
\hline Berlín & Bettemsteuer & $5 \%$ precio por pernoct. \\
\hline Bratislava & City Tax & $0,50-1,65 €$ \\
\hline Brujas & City Tax & $2 €$ \\
\hline Bruselas & City Tax & $2-7 €$ \\
\hline Bucarest & City Tax & $0,5 \%-5 \% €$ \\
\hline Budapest & City Tax & $0,25-1 €$ \\
\hline Dubrovnik & Sojourn Tax & $1-5 €$ \\
\hline Florencia & Contributo/Tassa/Imposta di Soggiorno & $2,5 €$ \\
\hline Gante & City Tax & 0,50 cent. $€$. \\
\hline La Valeta & Eco-tax & $1 €$ \\
\hline Lisboa & Taxa Municipal Turística & $0,60-1,27 €$ \\
\hline Llubljana & City Tax & $1-5 €$ \\
\hline Milán & Contributo/Tassa/Imposta di Soggiorno & $1-2 €$ \\
\hline Palma Mallorca & Impuesto de Turismo Sostenible & $0,65-1,5 €$ \\
\hline París & Taxe de Séjour & $1 €$ \\
\hline Praga & City Tax & $3-7 €$ \\
\hline Roma & Contributo/Tassa/Imposta di Soggiorno & $1-5 €$ \\
\hline Venecia & Contributo/Tassa/Imposta di Soggiorno & $0,15-2,18 €$ \\
\hline Viena & Beherbergungsbetreib & $0,30-0,60$ cent. $€$. \\
\hline Vilna & City Tax & $4 \%$ procio \\
\hline
\end{tabular}

*IEET: Impuesto de Estancias en Establecimientos Turísticos. Fuente: Elaboración propia (2016).

\subsection{Las tasas turísticas por pernoctación en España}

En España, la Ley General Tributaria ${ }^{1}$ que entró en vigor el 1 de julio del año 2004, define y diferencia los tributos (artículo 2.2). "Los tributos, cualquiera que sea su denominación, se clasifican en tasas, contribuciones especiales e impuestos: a) Tasas son los tributos cuyo hecho imponible consiste en la utilización privativa o el aprovechamiento especial del dominio público, la prestación de servicios o la realización de actividades en régimen de derecho público que se refieran, afecten o beneficien de modo particular al obligado tributario, cuando los servicios o actividades no sean de solicitud o recepción voluntaria para los obligados tributarios o no se presten o realicen por el sector privado. b) Contribuciones especiales son los tributos cuyo hecho imponible consiste en la obten-

1 Ley 58/2003, de 17 de diciembre, General Tributaria. Art. 2.2, en «BOE» núm. 302, de 18 de diciembre de 2003, páginas 44987-45065 (79 págs.). 
ción por el obligado tributario de un beneficio o de un aumento de valor de sus bienes como consecuencia de la realización de obras públicas o del establecimiento o ampliación de servicios públicos. c) Impuestos son los tributos exigidos sin contraprestación cuyo hecho imponible está constituido por negocios, actos o hechos que ponen de manifiesto la capacidad económica del contribuyente."

Atendiendo a dicha definición legal, y aunque se conozca en España vulgarmente como tasa turística, el citado gravamen tiene carácter de impuesto. Es así como se recoge en la legislación existente de las regiones que actualmente lo tienen implantado: Impuesto sobre las estancias en establecimientos turísticos (IEET). Y no es esta la única razón que lleva a contradicciones actualmente en España el tema de nuestra investigación. Actualmente hemos vuelto a un debate ya antiguo en torno a ellas. Debemos recordar que en el marco del turismo español del siglo XXI existen dos casos que han tenido una gran repercusión mediática en diferentes momentos históricos, las Islas Baleares y Cataluña. Disponemos de literatura especializada previa a la implantación de la primera tasa nacional (Bouazza, 2001; Dopazo, 2001; Gago \& Labandeira, 2001; Santamarta \& Castejón, 2001), y posteriores a la misma (Aguiló et al, 2005; Amer, 2003; Cantalops, 2004; García-Aranda, 2004; Mercado, 2003; Palmer \& Riera, 2003; Palmer et al, 2007). Así como estudios sobre sus posibles impactos y repercusiones en diferentes destinos nacionales (Adame, 2013; Font, 2012; Prados, 2001; Roca et al, 2004; Simancas, 2008) e internacionales (Ponjan \& Thirawa, 2016).

En el archipiélago balear recordamos dos etapas en torno al impuesto turístico. Una primera (2002-2003), en la que se estableció por primera vez el impuesto sobre las estancias en alojamientos turísticos, ecotasa. La idea, surgida por la fuerte presión turística que sufrió la región en la década de los años noventa del pasado siglo, tuvo una doble finalidad: hacer frente a los impactos negativos ocasionados por el turismo e incrementar la calidad de las islas como destino turístico. La situación de Baleares en aquel momento era insostenible. La fuerte demanda de agua, energía, así como de territorio hacía temer por la desvalorización del destino. "La producción de residuos sólidos urbanos es la más alta de España y el doble de la media nacional [...]; el consumo de energía se había disparado de forma alarmante en los últimos años (37\% entre 1993 y 1998); y la masificación y degradación de la costa balear, de los espacios turísticos y naturales y la pérdida de prestigio entre los visitantes" (Bouazza, 2001). Evitar una situación más grave requería de fondos adicionales de cara a paliar los efectos negativos de la actividad turística. La ecotasa supuso una recaudación de más de 80 millones de euros. Esta se dirigió a la conservación y mejora de los espacios verdes y recuperación del daño ambiental (ATIB, 2016; Vargas, 2016)

Sin embargo, debido a discrepancias entre la administración pública y el sector hotelero, la medida no prosperó y un año después de su implantación se retiró. Algunas de estas diferencias tenían que ver con que el impuesto era discriminatorio, pues solo lo tenían que abonar los turistas alojados en establecimientos turísticos reglados. Y, por otro lado, se aumentaba el coste de las vacaciones de los turistas en las islas, lo que se entendía como una amenaza para la competitividad de un destino muy consolidado aunque con indudables síntomas de agotamiento. 
En la segunda etapa, a partir del 1 de julio de 2016, la conocida ecotasa pasó a denominarse "impuesto al turismo sostenible". Esta reimplantación de un impuesto al turismo ha traído más enfrentamiento aún entre el sector hotelero y el Gobierno de Baleares, siendo uno de los principales motivos el destino de la recaudación por el citado concepto. Si bien el primer año irá dirigido a subsanar el gasto de la administración pública, la recaudación posterior debe ir a un Fondo para favorecer el turismo sostenible. Con el medio ambiente como punto de partida, la recientemente creada Comisión de Impulso del Turismo Sostenible se dirige a combatir una de las mayores preocupaciones del pueblo balear: el ciclo del agua (depuración, reutilización de aguas residuales, etc.). Por otro lado, la nueva Ley en la que esta nueva propuesta se enmarca, establece una clara diferencias respecto a la extinta ecotasa. Se trata de la regulación de las viviendas turísticas de alquiler, que deben cobrar el impuesto al turismo sostenible, así como las personas que viajen en cruceros (Ley 2/2016, de 30 de marzo, del impuesto sobre estancias turísticas en las Illes Balears y de medidas del impulso del turismo sostenible). Datos de la Agencia Tributaria balear recogen que el $85,4 \%$ de lo recaudado en los primeros meses lo ha aportado el sector hotelero. Dado que en este primer periodo no estaban obligados a cumplir con sus obligaciones fiscales los propietarios de las 9.110 viviendas turísticas regladas. Estas deberán hacerlo entre el 1 de mayo y el 30 de junio de 2017 (ATIB, 2016).

Por otro lado, el caso de Cataluña: en noviembre de 2012 la Generalitat implantó el "Impuesto sobre las estancias en establecimientos turísticos", denominado coloquialmente como tasa turística y recogido en la Ley 5/2012, de 20 de marzo, de medidas fiscales, financieras y administrativas y de creación del impuesto sobre las estancias en establecimientos turísticos. Sin embargo, el debate sobre la aplicación de la tasa viene de lejos; pero se podría decir que esta vez no ha generado la misma polémica que se desencadenó tiempo atrás (Font, 2012). El objetivo de la administración catalana con el establecimiento de este impuesto es mantener Cataluña como destino competitivo y de calidad. Esto se prevé lograr destinando la recaudación a la promoción turística de Cataluña, el impulso del turismo sostenible, responsable y de calidad, la protección, preservación, recuperación y mejora los recursos turísticos, el fomento, la creación y la mejora de los productos turísticos, y el desarrollo de infraestructuras relacionadas con el turismo (Decreto 161/2013, de 30 de abril, por el que se aprueba el Reglamento del Fondo para el fomento del turismo). Desde su implantación, tanto el número de visitantes como los ingresos generados por el impuesto han presentado un crecimiento positivo. En 2015 se recaudaron 43,5 millones de euros, un 5,48\% más que en 2014 . Y aunque los hoteles son la principal fuente de recaudación (79\%), las viviendas de uso turístico ingresaron un 38,6\% más que en 2014 (3,59 millones de euros), debido al esfuerzo realizado en la regulación de estos alojamientos (Hosteltur, 2016).

En el caso de la ciudad de Barcelona, el Ayuntamiento tiene como principal objetivo desarrollar nuevos servicios e infraestructuras que favorezcan la desconcentración territorial de los turistas, ayuden a repartir la riqueza que generan los visitantes entre todos los barrios y distritos y contribuyan a encajar el turismo con la vida diaria de los ciudadanos. Supone un coste al turista por noche de $0,75 €$ en hoteles de 1 a 3 estrellas, $1,25 €$ en hoteles de 4 estrellas y 2,50€ en hoteles de 5 estrellas. Queda limitada a un máximo de 7 noches y están exentos de pago los menores de 16 años. También los visi- 
tantes en crucero habrán de pagar 2,50€. Tras cuatro años de existencia del impuesto sobre las estancias en alojamientos hoteleros, actualmente se estudia crear un impuesto turístico que grave también a los excursionistas (Ayuntamiento Barcelona, 2016).

El caballo de batalla más reciente desde el ayuntamiento es conseguir gestionar el $100 \%$ de los ingresos generados por este concepto, pues hasta la actualidad la ciudad recibe solo una tercera parte. En el año 2015 generó 22,2 millones de euros, lo que suponía el 51\% del total de la recaudado en Catalunya (43,5 millones). A pesar de ello, las arcas municipales ingresaron 9,5 millones, correspondientes al 33\% de lo recaudado más una aportación extraordinaria de dos millones. Desde el gobierno local se considera insuficiente e injusto y se mantiene abierto el debate en la región. En la misma línea de lo que ha sucedido en estas regiones, en los últimos años se ha despertado en otras comunidades autónomas un interés creciente en la aplicación de tasas específicas que graven las actividades turísticas (Gago et al, 2009). Las polémicas más recientes se han dado en la Comunidad de Madrid, Galicia, el País Vasco (dos propuestas de estudio, 2013 y 2016), las Islas Canarias (desde 2013), Comunidad Valenciana (2016) y Andalucía (desde 2015, en Granada y Sevilla). Y es este debate en la capital andaluza quien suscita el interés de la investigación y justifica un estudio más a fondo.

\section{OBJETIVOS Y METODOLOGÍA}

El objetivo general de este estudio es conocer si en un destino turístico urbano como "Sevilla" es posible la implantación a corto plazo de una tasa que grave los alojamientos turísticos. Para ello se han establecido varios objetivos específicos:

- Analizar el comportamiento del destino Sevilla a través de la evolución turística experimentada en los últimos años (2008-2016), coincidiendo con la crisis económica y recuperación.

- Considerar la posible implantación de la tasa turística en la ciudad, a través de la información proporcionada por profesionales del sector turístico de la ciudad, y establecer el nivel de acuerdo existente en primera instancia entre ellos a escala local. Identificando los beneficios e inconvenientes que conllevaría la misma. Y conocer la opinión y valoración de los turistas que visitan el destino.

- Asimismo se pretende de manera transversal conocer las relaciones entre el sector público y privado y la posibilidad de consenso entre las escalas local y regional frente a la potencial implantación de una tasa turística. Y reflexionar sobre los posibles impactos de la misma en términos de sostenibilidad territorial y turística del destino.

Para abordar el estudio de los indicadores turísticos de la ciudad de Sevilla se han trabajado los Balances Anuales del Turismo y Estudios de Coyuntura de Sevilla entre los años 2008-2015. Y se han incorporado los datos más recientemente publicados por el Consorcio de Turismo (Ayuntamiento de Sevilla, 2017). Estos informes ofrecen información tanto cualitativa como cuantitativa en relación a la procedencia de los turistas, tipo de alojamiento utilizado, estancia media, grado de ocupación, motivación, satisfacción del turista, fidelidad de los mismos, etc. Junto a los anteriores, para conocer la demanda se ha trabajado con la Encuesta de Ocupación Hotelera (E.O.H) y la Encuesta de Ocupación en Apartamentos Turísticos (E.O.A.T.), elaboradas por el Instituto Nacio- 
nal de Estadística (INE, 2016) y a escala más detallada, por el Instituto de Estadística y Cartografía de Andalucía (IECA). Así como con los datos proporcionados por SAETA, Empresa Pública Turismo y Deporte de Andalucía a partir de los anteriores.

En lo que a fuentes primarias se refiere, se han realizado 14 entrevistas a informadores privilegiados con 10 preguntas abiertas. Representantes del sector turístico sevillano, que por su larga trayectoria profesional, juegan en la actualidad o han tenido en los últimos años un papel fundamental en la política turística de la ciudad y en la toma de decisiones. Otros, por su conocimiento en turismo receptivo y preferencias de la demanda turística; por la realización de labores de asesoramiento, mediación y comercialización, así como por el trato con turistas. Y en otros casos, por su experiencia en la elaboración de documentos estratégicos y en materia de planificación a diferentes escalas. Todos ellos pertenecen a diferentes subsectores turísticos de la ciudad de Sevilla, tanto del sector público como privado: 2 representantes de la Administración local sevillana, 2 representantes de la Asociación de Hoteles de Sevilla, 3 agencias de viaje (AAVV), 2 consultoras de turismo y planificación turística y 5 hoteles de diferente categoría.

Paralelamente se ha realizado un cuestionario para conocer la opinión de los turistas que visitan Sevilla. Junto a su conocimiento sobre las tasas turísticas y cómo afectaría en sus preferencias de destino, es interesante conocer su valoración. Para ello se ha escogido una muestra de 100 turistas (50\% nacionales-50\% internacionales) a los que se les ha pasado un cuestionario de 20 preguntas, con respuestas cerradas en una "escala Likert" del 1 al 4, donde 1 es Totalmente en desacuerdo y 4, Totalmente de acuerdo. Se ha escogido este intervalo para que no exista una respuesta intermedia y los encuestados se vean obligados a posicionarse en una postura clara. Estos cuestionarios se han realizado en diferentes puntos calientes del centro histórico de la ciudad: Plaza del Triunfo, Calle Mateos Gago, Avenida de Constitución, Ayuntamiento, Plaza de España, Plaza de El Salvador y Plaza de la Encarnación-Setas, coincidiendo con las fechas de mayor afluencia turística. Tanto los cuestionarios como las entrevistas se han realizado en 2016, entre los meses de abril-mayo y octubre, respectivamente.

\section{LA EVOLUCIÓN DEL TURISMO EN SEVILLA}

Sevilla es uno de los destinos turísticos urbanos más visitados de España. En al año 2015 la ciudad registró máximos históricos en volumen de llegada de turistas. Alcanzó 2.307.018 de viajeros alojados en establecimientos hoteleros (un 3,38\% del total nacional). Estos datos posicionaron a Sevilla como tercer destino urbano nacional, tras Madrid y Barcelona (8.885.778 y 7.093.372 de viajeros, respectivamente). Mientras que en lo que a pernoctaciones se refiere y a pesar de los buenos datos obtenidos se mantiene en un decimotercer lugar (4.604.780) según datos del INE (2016) a través de la Encuesta de Ocupación Hotelera (E.O.H).

\subsection{Datos 2008-2015: crisis y recuperación}

A continuación se muestra la evolución del turismo entre los años 2008-2015 desde el comienzo de la crisis económica. Es en este periodo 2008-2015 cuando se producen las mayores turbulencias en el crecimiento del turismo sevillano del siglo XXI, manifestándose en dos periodos de recesión: 2008-2009 y 2011-2012. 


\section{Figura 2 \\ EVOLUCIÓN DE LA LLEGADA DE TURISTAS Y PERNOCTACIONES}

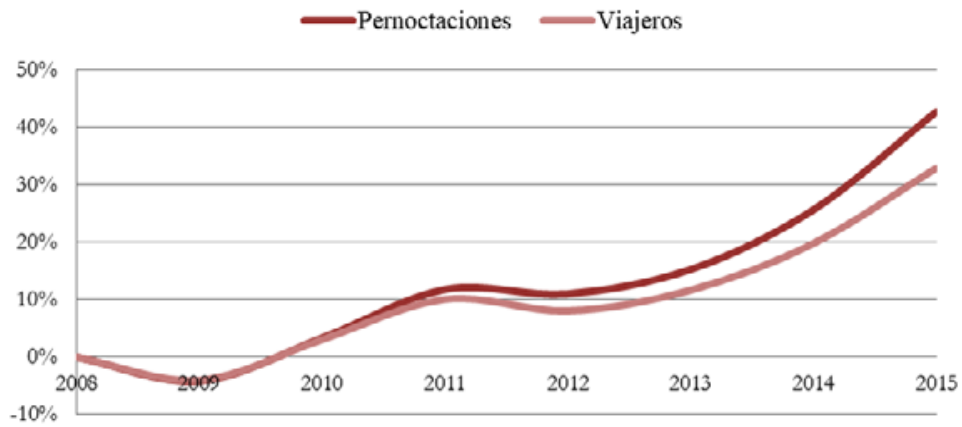

Fuente: Encuesta de Ocupación Hotelera (INE, 2016). Elaboración propia.

Tanto la llegada de turistas como el número de pernoctaciones hoteleras, se ve afectada por la coyuntura económica con unos porcentajes de decrecimiento de más del $4 \%$ para el primer periodo, de 2 puntos para los viajeros y $0,8 \%$ para las pernoctaciones en el segundo periodo. Sin embargo, la variación más significativa se da entre los años 2014 y 2015 y, en este caso, ha sido positiva. Un crecimiento de más del $13 \%$ en llegadas de viajeros, y cerca de un $17 \%$ en el número de pernoctaciones hoteleras, dieron lugar a que 2015 se consolidara con el mejor registro turístico para la ciudad de Sevilla. El número de viajeros en 2015 aumentó en algo más de un 30\% respecto al año 2008 (figura 2) y paralelamente, el número de plazas hoteleras también creció en torno a un $12 \%$. Con este contexto, se analiza en primer lugar la evolución y procedencia de los viajeros, así como su distribución por tipo de alojamiento, la estancia media y el grado de satisfacción de los turistas que visitan la ciudad.

Figura 3

\section{VOLUMEN DE TURISTAS}

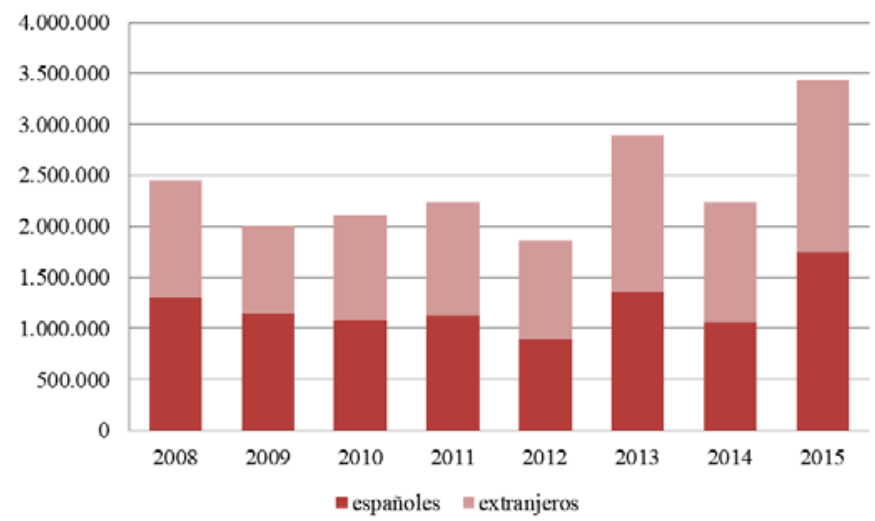

Fuente: Elaboración propia. Datos E.O.H, INE 2016. 


\section{Figura 4 \\ TURISTAS SEGÚN PROCEDENCIA}

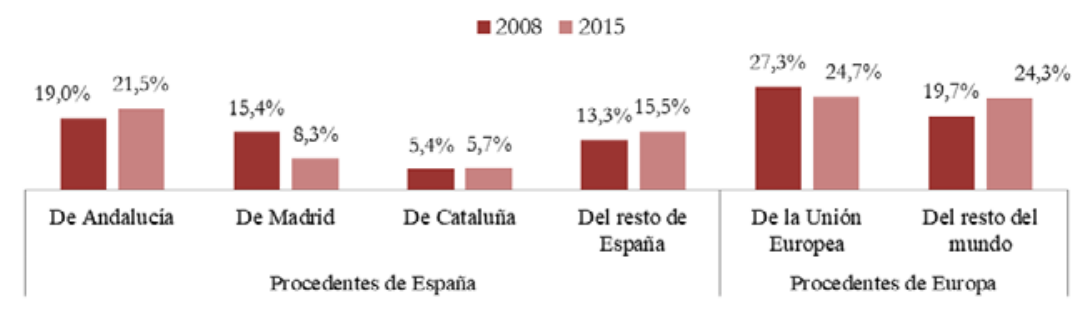

Fuente: Elaboración propia. Datos E.O.H, INE 2016.

La afluencia de turistas procedentes del extranjero (figura 4) en el periodo 2008-2015 ha experimentado un crecimiento superior al de los nacionales. Destacando el volumen de turistas que proviene de países exteriores a la Unión Europea, principalmente de EEUU, dato que se ha venido consolidando e incrementando año tras año. En lo que a establecimientos hoteleros se refiere, la oferta turística sevillana está compuesta por 234 con un total de 20.404 plazas, según el Registro de Turismo de Andalucía, 2015. En volumen de establecimientos destacan los hostales y pensiones, situados en su mayoría en el casco histórico de la ciudad. Sin embargo, el mayor volumen de plazas se concentra en los hoteles de cuatro estrellas, con casi el $60 \%$ de la oferta, seguidos a gran distancia de los de 3 estrellas (15\% del total). En relación a los establecimientos de mayor categoría, ofrecen un total de 1.456 plazas, que se traduce en el $7 \%$ de la oferta de plazas hoteleras. En 2015, la capital andaluza contaba con el 68,1\% del total de las plazas registradas en la provincia de Sevilla, algo más de un punto superior a la misma variable en 2008. Estos datos no incluyen la oferta existente en los municipios de la aglomeración urbana, lo que evidencia la importancia de la urbe en la provincia. A nivel andaluz, representa el 7,2\% de las plazas ofertadas por la Comunidad Autónoma, seguida a gran distancia de Granada (14.111 plazas) y Málaga (9.948 plazas).

Tabla 2

DISTRIBUCIÓN DE PLAZAS TURÍSTICAS HOTELERAS EN SEVILLA (2015)

\begin{tabular}{|c|c|c|c|c|c|c|c|}
\hline Categoría & $1 *$ & $2 *$ & $3 *$ & $4 *$ & $5 *$ & Otros & TOTAL \\
\hline $\mathrm{N}^{\circ}$ Establecimientos & 45 & 27 & 34 & 51 & 5 & 72 & 234 \\
\hline $\mathrm{N}^{\circ}$ de Plazas & 1.260 & 890 & 3.041 & 11.974 & 1.456 & 1.783 & 20.404 \\
\hline
\end{tabular}

Fuente: Elaboración propia. Datos del Registro de Turismo Andalucía, 2016.

En relación a la evolución de la ocupación de plazas hoteleras (figura 5), se ha escogido la misma serie de años para observar la influencia de la crisis económica en el sector hotelero durante los tres ámbitos de referencia. A simple vista se aprecia como la situación en oferta de plazas ha aumentado respecto a 2008, sin embargo se dan una serie de etapas 
de estancamiento e incluso de recesión. A escala local pueden diferenciarse cuatro etapas: una comprendida entre los años 2008 y 2012 (los peores años de la crisis), donde registra un desarrollo progresivo año tras año con un crecimiento entre los dos años del $10 \%$. Y tras un año de recesión, en 2014 vuelve a experimentar un crecimiento del número de plazas en cerca del 3\%. El último año, se produce un periodo con valores negativos algo más acentuados que los acaecidos entre 2012 y 2013.

En cuanto a la distribución por tipo de alojamiento, aunque el número de viajeros y pernoctaciones ha aumentado en el periodo de estudio, el porcentaje de turistas alojados en establecimientos hoteleros ha disminuido considerablemente (un 4\%). Esta dinámica tiene su principal causa en la irrupción del fenómeno mal conocido como turismo colaborativo. La nueva figura de viviendas con fines turísticos (VFT), sin regular hasta febrero de 2016 en Andalucía, está registrando un crecimiento progresivo y muy fuerte debido principalmente al factor precio.

La variable que ha centrado el foco de atención en estos últimos años para la política turística hispalense ha sido la estancia media. Variable que difiere considerablemente en función del tipo de establecimiento. Mientras que para los hoteles se sitúa en torno a 2 noches, los apartamentos turísticos registran una estancia media de 2,71 noches. El aumento de la estancia media así como el del número de pernoctaciones en los establecimientos hoteleros, han dado lugar a que el grado de ocupación por plaza haya crecido alrededor de un $8 \%$ en los últimos 7 años.

\section{Figura 5}

\section{EVOLUCIÓN DE PLAZAS HOTELERAS}

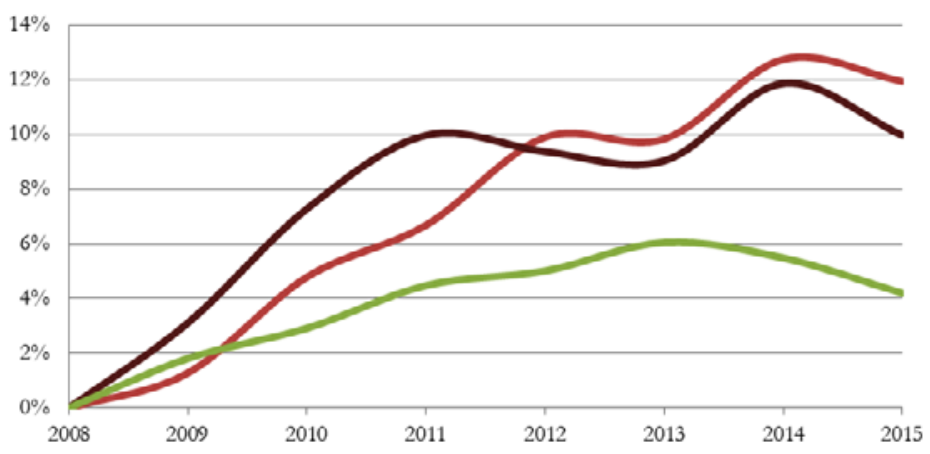

Fuente: Elaboración propia. Datos del Registro de Turismo de Andalucía (SAETA, 2016).

Por último, para conocer la calificación del destino Sevilla por parte de los turistas atendemos a la información proporcionada por el Balance anual de Turismo que elabora el Consorcio de Turismo de Sevilla (2016). Dicho estudio, muestra como la opinión sobre el sector turístico ha registrado un ascenso de prácticamente 0,5 puntos en el periodo 2008 2015, situándose, en una escala de 1 Poco Satisfactorio - 5 Muy Satisfactorio, en 4,97. El crecimiento más significativo se da en el apartado Ocio y Diversiones $(+0,76)$, seguido de la Restauración $(+0,69)$ y Alojamiento $(+0,47)$. 


\section{Figura 6 \\ CALIFICACIÓN DEL VIAJE}

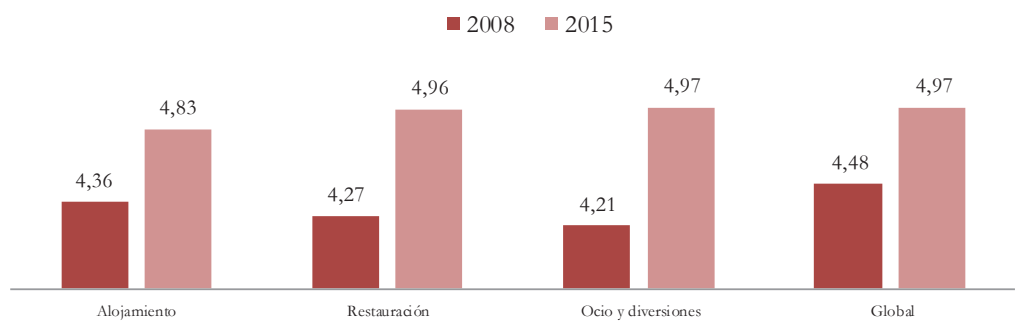

Fuente: Elaboración propia. Datos del Consorcio de Turismo de Sevilla (2016).

\subsection{Logros históricos y nuevos retos a partir de 2016}

Para finalizar con esta evolución de los datos del destino es oportuno incluir los más recientes, publicados por el Consorcio de Turismo en febrero de 2017 (Ayuntamiento de Sevilla, 2017). Y son estos los que señalan que en 2016 aún fueron mayores los logros del destino tanto en ocupación media mensual como en el total del ejercicio.

En el conjunto del año, la suma de datos hoteleros a los de apartamentos turísticos, muestran una clara y ventajosa diferencia respecto a los anteriores, ya analizados. Lo que posiciona la última campaña como la mejor de la historia de Sevilla. Se alojaron 2,7 millones de viajeros y realizaron 5,5 millones de pernoctaciones regladas. Un 10,24\% superior en viajeros y un $9,04 \%$ en pernoctaciones con respecto al año 2015.

Aunque la estancia media se sigue manteniendo en 2016 en torno a los dos días, concretamente en 1,97 días, la oferta ha seguido creciendo: se abrieron un 4,31\% más de establecimientos, un total de 10.798 habitaciones con disponibilidad de 21.660 plazas, un $1,18 \%$ más que en diciembre de 2015.

El año 2016 cerró su ejercicio en la capital andaluza con 2,5 millones de viajeros alojados en hoteles, un 9,04\% superior al 2015 (E.O.H, INE 2017); 5 millones de pernoctaciones, lo que representa un 7,75\% más que en el pasado año; una estancia media de 1,97 días, y un aumento medio del número de personal ocupado del 2,10\%. Los datos acumulados revelan además que registró el mejor primer semestre en la historia en la capital, según las series homogéneas de cifras disponibles. Y por otro lado, los meses de verano registraron cifras de ocupación más elevadas, lo que revela la creciente desestacionalización del turismo en la capital (ejemplo de ello es el 79\% de ocupación durante el mes de junio).

Al mismo tiempo, los datos de la E.O.A.T (INE, 2017) muestran que se han alojado en los apartamentos turísticos durante el 2016, 18.739 viajeros, lo que representa un $49,66 \%$ más que en 2015 . Estos han producido 49.301 pernoctaciones $(47,82 \%$ superior). Podemos destacar por tanto, el incremento en la oferta de este sector, tanto en apartamentos (23,21\% superior), como en plazas ( $23,59 \%$ más), a lo que hay que sumar un $11,48 \%$ más de personal empleado. La aportación de este sector reglado del turismo ha favorecido el alojamiento de 195.000 viajeros y 392.000 pernoctaciones. 
Frente a estos datos, la dificultad estriba en cuantificar y medir los impactos del resto de alojamientos no reglados con fines turísticos que existen actualmente en la ciudad y que cada vez son más numerosos, impulsados por nuevos modelos y canales de comercialización P2P (portales web de alquiler como airbnb, homeaway o windu, entre otros). $\mathrm{Y}$ es que mientras que el alojamiento regulado ha sido tradicionalmente objeto de estudio exhaustivo por parte de las instituciones estadísticas (estatal, autonómicas y municipal), no existe una información clara y objetiva sobre la oferta de alojamiento turístico en viviendas de alquiler ni para Sevilla ni para ningún otro destino urbano español, tal y como queda recogido en un estudio reciente elaborado por Ernest \& Young, publicado por Exceltur. Se trata de una discusión global ya presente en los principales destinos turísticos del mundo. Muchos de los cuales disponen de marcos normativos (al que ya se ha sumado Andalucía), para tratar de limitar los impactos desfavorables de este fenómeno, e incluso prohibir de facto ese tipo de alojamientos, por considerarlos perjudiciales para el desarrollo de su modelo turístico y/o de convivencia ciudadana (Exceltur, 2015).

En España, según el informe Monitor de la Competitividad Turística de los destinos urbanos españoles (UrbanTUR 2016), el número de plazas en viviendas ofertadas en las principales plataformas como alojamientos turísticos en las 22 ciudades analizadas se sitúa en 362.493. Ello supone un aumento del 88,3\% respecto al número existente en 2015 y del $1633 \%$ en relación con 2012. Tan desproporcionado como insostenible (Exceltur, 2017). Y en el destino que nos ocupa, se ha estimado desde la administración local hispalense que existen más de 20.000 plazas fuera de todo control legal. De ellas, y tras la entrada en vigor del Decreto 28/2016, de 2 de febrero de las viviendas con fines turísticos (sustentado en la Ley 13/2011, de 23 de diciembre del Turismo de Andalucía), se han registrado 1.700 viviendas, lo que supone unas 7.000 plazas legalizadas.

$\mathrm{Su}$ control y su conocimiento es prioritario de cara a una adecuada toma de decisiones tanto desde la administración como por parte del empresariado turístico. Y éstas han de basarse en información fidedigna y puntual, lo que hace necesario ampliar la sistemática de recogida y análisis de los datos referentes a esta oferta alegal. Los impactos sociales y económicos que se derivan de esta problemática turística ya se están haciendo notar en otros destinos urbanos españoles. Tal es el caso de la ciudad de Barcelona, con una oferta sobredimensionada a razón del nuevo modelo alojativo y una demanda tan excesiva como peligrosa para su sistema turístico local: generadora de impactos territoriales, ambientales, urbanísticos, inmobiliarios, e incluso enfrentamientos sociales de residentes frente a la hotelización de viviendas particulares. Un modelo turístico este de la capital catalana que, si bien reporta cuantiosos beneficios a las arcas municipales, se aleja de la calidad turística y de la tan ansiada sostenibilidad territorial. Si bien, el ya comentado UrbanTUR 2016 la posiciona como el destino urbano más competitivo de España.

\section{RESULTADOS}

Para poder obtener y contrastar las opiniones de informadores privilegiados del sector turístico sevillano, así como la de los turistas que visitan la ciudad, se optó por la realización de entrevistas (14) y encuestas (100) respectivamente. En relación a los resultados obtenidos de los primeros, se ponen de manifiesto varias cuestiones: 
Desde la Asociación de Hoteles de Sevilla y Provincia (AHS) la postura frente a una posible tasa es clara: "ya se está contribuyendo de forma notable al sostenimiento del sistema turístico con los impuestos que se pagan a distintos niveles administrativos. $Y$ ello sin contar con la contribución que hacen las propias empresas turísticas de la ciudad para promocionar el destino, a través de sus departamentos comerciales. "Sin ninguna duda, son las empresas que más invierten en la promoción del destino en su conjunto, porque nuestros alojamientos no se venden, si no se vende el destino”. Asimismo, no están de acuerdo en que solo se grave a establecimientos turísticos de alojamiento, y sí a cualquier actividad turística. "Los hoteleros ya están absolutamente agobiados por la presión fiscal en estos momentos". Tanto desde este mismo sector hotelero como desde las Agencias de viajes (AAVV) se argumenta que "el impuesto es discriminatorio, debido a que sólo contribuyen los visitantes que se hospeden en alojamientos turísticos reglados, favoreciendo así el crecimiento de las viviendas de alquiler". Asimismo, "el turista es muy sensible a las pequeñas variaciones en el precio y a largo plazo tendrán un efecto negativo". Añaden las AAVV que el impuesto "debería gravar a la oferta hotelera y extra hotelera, incluyendo a las viviendas con fines turísticos".

Desde las empresas Consultoras la opinión es diferente, entendiendo que "la tasa turística puede ser un buen instrumento de promoción turística y, como tal, debería gravar a todas las actividades que se vieran beneficiadas de esta medida, y no solo al sector hotelero". Del mismo modo, se hace hincapié en que "sería beneficioso vender la tasa sobre la base de pertenencia de un club de ciudades élites en turismo." Sin embargo, apuestan por realizar previamente un estudio de viabilidad económica. Y de alcanzarse un consenso en el sector, apoyarían una propuesta formal al gobierno autonómico.

Una de las cuestiones que más preocupa al sector turístico en su conjunto es la recaudación del impuesto ¿quién debe gestionarla y dónde debe dirigirse la misma? Por ello, en la entrevista realizada a estos profesionales se incluye una pregunta acerca de cuál debería ser el destino de la misma en el supuesto caso de que se estableciera en Sevilla. Y quién debe ser responsable de esta gestión. En este sentido, el sector hotelero "aplicaría la tasa única y exclusivamente teniendo la certeza y la seguridad de que se administre de forma clara y adecuada y se aprueben las cuentas de forma conjunta con los organismos que la gestionan, siendo este órgano gestor una representación del sector turístico con un interventor", y el 50\% dejaría su recaudación al gobierno local. Otro 50\% apuesta por la compartida con el gobierno regional. En lo que al destino de los fondos recaudados se refiere, de los 14 entrevistados, 10 inciden en la necesidad de promoción turística del destino. Frente a 4 que apuestan porque "su recaudación debería destinarse a sufragar gastos públicos que afecten y/o se vean afectados por el turismo de diferente tipo (limpieza, seguridad,...)". Ante la posibilidad a que pudiera formularse una propuesta formal al gobierno andaluz, esta sería apoyada, de existir consenso, por todos los agentes entrevistados. Los mayores desencuentros enfrentan al gobierno local y regional, sencillamente, porque la Junta de Andalucía ha decidido, dado que es su competencia, la no aplicación de la tasa turística en el territorio andaluz. Las razones que conocemos son retóricas: debe aplicarse a todo el territorio y no a Sevilla como excepción (el cansino complejo de la excepcionalidad sevillana) y más retórico aún, la política regional andaluza en turismo debe caminar en la senda de la cualificación turística, destinos de calidad, y no en el de la fiscalidad. 
De todo ello se deduce que entre los principales agentes turísticos y el gobierno local y regional no existe consenso en relación al impuesto. Y se mantienen dos posturas enfrentadas en torno a su implantación. Por otro lado, las preguntas abiertas han permitido detectar el problema que genera mayor inquietud y controversia dentro del sector y que ha sido recurrente objeto de queja por parte de todos los entrevistados. Muy relacionado con la tasa, plantean la obligatoria regulación de las viviendas de alquiler con fines turísticos, exentas de obligaciones fiscales por ser alegales. Y plantean la posibilidad de llegar a un acuerdo con portales web de alquiler para aplicar la misma tasa sobre estas, como ya se hace en otras ciudades europeas como Florencia o Lisboa. Como ya se comentara en el apartado anterior, el enorme potencial de crecimiento de esa oferta alojativa constituye un peligroso desafío para muchos destinos turísticos españoles. Y Sevilla, desde su posicionamiento competitivo en el mercado nacional, no sólo no está ajena a esta realidad, sino que se opone. Es por ello que desde el empresariado turístico, no exclusivamente hotelero, se exigen medidas y actuaciones periódicas de inspección y control. Una regulación imprescindible y urgente, previa a cualquier debate sobre tasas.

El otro conjunto al que nos hemos asomado de forma somera en este estudio (a través de encuestas) han sido los turistas, como contribuyentes potenciales al tributo. Si bien, no eran estos nuestro objeto central de análisis. Los profesionales entrevistados les conceden un papel secundario y, por tanto, su opinión no es relevante en la toma de decisiones. Aun así, entre los objetivos de este trabajo estaba aproximarnos a la opinión de los turistas que visitan el destino para detectar en qué medida y de qué forma podría afectar la aplicación del impuesto a la demanda turística. Y por otro lado, su conocimiento sobre el mismo. De los resultados obtenidos por el cuestionario, y a pesar de lo reducido de la muestra, se extraen diferentes conclusiones, entre las que cabe destacar:

De los turistas españoles, el $33 \%$ estaba familiarizado con la tasa turística y el $38 \%$ conocía los aspectos más básicos del tributo. Sólo un $15 \%$ afirmaba conocer a fondo esta tasa. Frente a ellos, los turistas extranjeros están familiarizados con el pago de tasas en sus viajes $(55 \%)$ y lo asumen como algo frecuente.

La existencia de una tasa turística de alojamiento en un destino urbano de similares características a Sevilla no es un motivo suficiente para no visitarlo (70\% de los encuestados).

El 98\% del total de los encuestados estarían interesados en conocer donde irían dirigidos esos ingresos y una explicación clara del impuesto y su forma de recaudación.

Un $88 \%$ de los encuestados coincidiría con la iniciativa del sector turístico de destinar la recaudación del impuesto a la promoción exterior del destino.

Por otro lado, el $82 \%$ de los encuestados estaría a favor de establecer excepciones de cobro a turistas menores de 16 años, estancias máximas de 4 noches, turistas nacionales de negocios, etc.

Al 58\% de los turistas encuestados no le importaría pagar entre 0,50 y 2 euros en función de la categoría del alojamiento. Sin embargo, el $42 \%$ restante estaría en contra de contribuir con cualquier cantidad extra sobre la tarifa del alojamiento.

Los resultados obtenidos en relación a favor o en contra de la tasa turística han sido curiosamente equilibrados: $50 \%$ muestra su aprobación y $50 \%$ se muestra contrario. Además el $75 \%$ de los encuestados volvería a visitar de nuevo Sevilla, con o sin tributos turísticos por pernoctación. 
Por tanto, y aunque a priori y a corto plazo la demanda no parece preocupada por la posibilidad de pagar la tasa, por lo que se deriva de las opiniones profesionales sí que podría suponer daños en el destino a medio y largo plazo, viéndose perjudicados tanto el sector hotelero como los comercios locales. Según este estudio, el $25 \%$ de los turistas no repetirían el destino por esta razón. Lo que merecería sin duda un estudio más en profundidad y con una muestra mucho más amplia y variada.

\section{CONCLUSIONES}

Tres consideraciones que se hallan en la ecuación de sostenibilidad versus fiscalidad turística:

1) Los destinos urbanos europeos más demandados, por obvia relación oferta/demanda, son los que más cobran al turista por pernoctar en sus hoteles: Amsterdam, Roma, Venecia, Berlín o Barcelona, ¿son buenos referentes de sostenibilidad turística?, ¿y en cuanto a la gestión de destinos?

2) Quizás en términos de ingresos (PIB) sí lo sean, en el corto y medio plazo. Pero no es garante de calidad, si previamente no se solucionan otros problemas de alegalidad e ilegalidad alojativa generadores de impactos territoriales de difícil solución en el largo plazo: masificación, pérdida de identidad, burbujas inmobiliarias, gentrificación, enfrentamientos sociales, e incluso aversión al turista por parte de unos residentes que padecen sus consecuencias.

3) Incrementar el consumo de los no residentes y la estancia media en las ciudad genera beneficios obvios a las arcas municipales, y la imposición de tasas extras multiplicaría sus efectos en términos cuantitativos. Pero, ¿mejoran por sí mismas la calidad del turista que nos visita, y la calidad de vida de los residentes?

A la luz de los resultados de esta investigación, la introducción de las tasas no parece ser un hecho inminente en la ciudad. De momento no existe el consenso necesario, ni dentro del sector ni a nivel político (local-regional). No es una prioridad. Y no lo es, porque no genera confianza entre los hoteleros, que encuentran en la competencia desleal su principal enemigo. No lo es, porque en destinos urbanos de referencia internacional, las tasas conviven con eco etiquetas y certificaciones de calidad que no han conseguido frenar la masificación turística. Tampoco lo es, porque nuestros destinos top nacionales están evidenciando debilidades que hacen peligrar un modelo aparentemente consolidado y exitoso en nuestro país. Y hoy por hoy, no son el espejo donde Sevilla quiere mirarse, ni esa es la imagen de destino que quiere mostrar al turismo. Por otro lado, las tasas no han demostrado ser en sí mismas ningún garante de calidad. Ni en los destinos, ni en los turistas que lo visitan.

Lo que sí es una prioridad para la ciudad, es responder de manera rápida a los nuevos retos que plantea la demanda, cada vez más joven e internacional, y con preferencias y maneras de bajo coste. De forma contundente, al exponencial y desordenado crecimiento de la oferta de alojamiento intermediada por plataformas P2P. Y de manera responsable, respecto a los estándares de calidad y sostenibilidad territorial por el que apuesta como destino turístico. 
En efecto, la sostenibilidad del turismo, entendiéndose el término en el sentido de oportunidad económica y ambiental, de residentes y de no residentes, se ha convertido en un objeto clave para el desarrollo propio del sector. Las tasas turísticas no tienen que coadyuvar necesariamente en este rol; pero como todo en las relaciones público-privadas pueden ejercer de elemento de confianza si se gestionan para un objetivo finalista acordado. La propia maquinaria de recaudación ex novo de la tasa turística es parte de los costes que es necesario imputar. La naturaleza jurídica de las tasas provoca que las mismas deban tener en su recaudación un objetivo finalista bien definido. La evolución viene siendo en esta materia difusa y alcanza últimamente más a confortar a la población residente de los impactos turísticos, que a la mejora del paisaje urbano turístico; más allá de lo clásico: como aportación a la promoción turística. Que también habrá que debatir en su efectividad real, en tiempos de turismo individual, métodos indirectos de promoción (tipo Film Commissión), nuevas formas de alojamiento, burbuja hotelera e intermediación TIC de la demanda.

En Sevilla, para adelantarnos a acontecimientos indeseables (se debate ahora mismo sobre la proliferación de veladores en la vía pública), sería procedente que los procesos de fiscalidad turística se encaminen a la finalidad estratégica de la sostenibilidad. La ciudad en los últimos tres años ha crecido en un ritmo de interés compuesto del $10 \%$ en pernoctaciones. En 2016 se produjeron un 50\% más que en 2010. Si esto no es boom se le parece muchísimo. Dicho de otra forma, 15 años para pasar de tres a cuatro millones de pernoctaciones y sólo tres para crecer de cuatro a cinco millones. En este punto es donde está el sitio de la tasa turística, pero también en el de renovar su propio concepto: también para la vivienda turística y para excursionistas y visitantes sin pernoctación. Hosteleros, restauración y agencias receptivas y de oferta complementaria, y como no el comercio dedicado al turista, deberían colaborar en la novación de la tasa. Pensamos que no es de recibo cargarla toda hacia el sector hotelero.

Ahora se discute en Sevilla sobre la ampliación de la peatonalización del centro -que ha sido muy colaboradora del mentado boom turístico- en dos calles saturadas de turismo (Mateos Gago y en menor medida, calle Betis). Estos cambios en el paisaje urbano turístico que afectan al residente y también a la calidad de vida del turista, deben ser el objeto de otra forma de ver la tasa turística: de forma sostenible. En resumidas cuentas, la fiscalidad turística para reajustar los procesos de saturación, debería basarse en el consumo de los no residentes mediante su aplicación en los servicios turísticos, más allá del alojamiento hotelero. El caso de Sevilla puede ser un excelente laboratorio si se ponen en marcha mecanismos de colaboración público-privada y se desarrollan acciones finalistas en la mejora del paisaje urbano y en la confortabilidad tanto de los residentes como de los que no lo son.

\section{BIBLIOGRAFÍA}

ABEYRATNE, I. (1993): «Air transport tax and its consequences on tourism», Annals of Tourism Research, vol. 20 (3), 450-460.

AGUILÓ, E., RIERA, A. y ROSELLÓ, J. (2005): «The short-term price effect of a tourist tax through a dynamic demand model: The case of the Balearic Islands», Tourism Management, vol. 26 (3), 359-365. 
ADAME MARTÍNEZ, F. (2013): «Turismo y financiación municipal: estudio sobre posibles nuevos Tributos Locales vinculados al turismo», Tributos Locales, n 112, 13-61. AMER FERNÁNDEZ, J. (2003): «Empresariado hotelero e implementación de un impuesto turístico: el caso de la ecotasa» en Baleares», Cuadernos de Turismo, $\mathrm{n}^{\circ} 12$, $165-178$.

ASHWORTH, G. y PAGE, S.J (2011): «Urban tourism research: Recent progress and current paradoxes», Tourism Management, vol. 32 (1), 1-15.

AYUNTAMIENTO DE SEVILLA (2015): Balance anual de 2008-2015 del Turismo de Sevilla. Indicadores Turísticos de la Ciudad de Sevilla. Consorcio de Turismo de Sevilla.

AYUNTAMIENTO DE SEVILLA (2017): Turismo de Sevilla. El Sistema de Información Turística de la ciudad de Sevilla, febrero 2017. Consorcio de Turismo de Sevilla.

BIRD, R. (1992): «Taxing Tourism in Developing Countries», World Development, $\mathrm{n}^{\circ}$ 20, 1145-1158.

BOKOBO, S. (1999): «La fiscalidad de las prestaciones turísticas. La posibilidad de que las Comunidades Autónomas establezcan la tasa turística», Civitas. Revista Española de Derecho Financiero, vol. 102, 243-260.

BONHAM, C., FUJII, E., IM, E., y MAK, J. (1991): «The impact of hotel room tax: An uninterrupted time series approach», National Tax Journal, vol. 45 (2), 433-441.

BOUAZZA, O. (2001): «La ecotasa balear: un ejemplo de situación de necesidad a la que nunca debió llegarse», Estudios Geográficos, nº 245, 737-744.

CANALIS, X. (2014): Túnez implanta una tasa turística. Hosteltur. Disponible en: http:// www.hosteltur.com/174630_tunez-implanta-tasa-turistica-13-euros.html

CANTALLOPS, A.S. (2004): «Policies supporting sustainable tourism development in the Balearic Islands: The Ecotax», Anatolia, vol. 15 (1), 39-56.

CROTTS, J.C. y MCGILL, G.A. (1994): «Compliance with Local Option Lodging Taxes: Theory and Measurement Strategies», Journal of Travel and Tourism Marketing, Vol. 3, 51-83.

DALEY, M (2017): Options for a tourism levy for London. London Finance Commission. Working Paper 83. GLAECONOMIC. London.

DECRETO 161/2013, de 30 de abril, por el que se aprueba el Reglamento del Fondo para el fomento del turismo. Diario Oficial de la Generalidad de Cataluña, de 3 de mayo de 2013, núm. 6368, 1-5.

DECRETO 28/2016, de 2 de febrero, de las viviendas con fines turísticos y de modificación del Decreto 194/2010, de 20 de abril, de establecimientos de apartamentos turísticos. Boletín Oficial de la Junta de Andalucía, de 11 de febrero de 2016, núm. 28, pp. 66-74.

DOPAZO, P. (2001): «Los tributos de carácter parafiscal: la nueva ecotasa balear», Derecho y Medio Ambiente, Revista Jurídica para el Desarrollo Sostenible, $\mathrm{n}^{\circ}$ 7, 9-30.

DURBARRY, R. y SINCLAIR, M.T. (2001): Tourism Taxation in the UK, Report for the Tourism Action Group, Confederation of British Industry.

ETOA (2016): Policy/Regulation and taxation/Tourist taxes. European Tour Operators Association (ETOA). Londres. Disponible en: http://www.etoa.org/policy/regulationand-taxation 
EXCELTUR. (2015): Alojamiento turístico en viviendas de alquiler. Ernst \& Young. Tourism \& Law abogados, pp. 104.

EXCELTUR. (2017): UrbanTUR 2016. Monitor de Competitividad Turística de Ciudades. Disponible: http://www.exceltur.org/wp-content/uploads/2017/05/URBANTUR2016-240516-documento.pdf

FISH, M. (1982): «Taxing international tourism in West Africa», Annals of Tourism Research, vol. 9 (1), 91-103.

FONT, X. (2012): «El finançament del turisme als municipis i la taxa turística a Catalunya». P3T, Journal of Public Policies and Territories, vol. 1 (3), pp. 30-38.

FRANCO, J.F. (1996): «Posibles repercusiones para España de la implantación de la ecotasa». Economía Industrial, no $310,13-18$.

FUJI, E., KHALED, M. y MAK, J. (1985): «The Exportability of Hotel Occupancy and Other Tourist Taxes», National Tax Journal, Vol. 38 (2), 169-177.

GAGO, A. y LABANDEIRA, X. (2001): «Turismo y fiscalidad ambiental: análisis comparado del impuesto eco-turístico balear», VIII Encuentro de Economía Pública: Cáceres, febrero de 2001.

GAGO, A. Y LABANDEIRA, X., PICOS, F. y RODRÍGUEZ, M. (2009): «Specific and general taxation of tourism activities. Evidence from Spain», Tourism Management, vol. 30 (3), 381-392.

GARCÍA ARANDA, E. (2004): «La Imposición sobre el Turismo en España: Principio y Fin de la Ecotasa Balear», Revista Aranzadi de Derecho Ambiental. Univ. Rey Juan Carlos, Madrid, España.

GARROD, B. (2004): Tourism management: managing for change, by Stephen J. Page. Butterworth-Heinemann, Oxford, 2003. $381 \mathrm{pp.}$

GOOROOCHURN, N. y SINCLAIR, M. (2005): «Economics of tourism taxation. Evidence from Mauritius», Annals of Tourism Research, vol. 32 (2), 478-498.

GOOROOCHURN, N. (2009): «Optimal commodity taxation in the presence of tourists», International Economic Journal, vol. 23, 197-209.

GUNN, C.A. (2002): Tourism planning: basis concepts cases. Routledge. London.

HALL, M. (2008): Tourism Planning: Policies, Processes \& Relationships. Pearson, Prentice Hall. London. $1^{\text {a }}$ edición, 2000.

HIEMSTRA, S. e ISMAIL, J.A. (1992): «Analysis of Room Taxes Levied on the Lodging Industry», Journal of Travel Research, vol. 31 (1), 42-49.

HIEMSTRA, S. e ISMAIL, J.A. (2001): «Research Note to Incidence of the Impacts of Room Taxes on the Lodging Industry», Revision to Article Published in Spring 1993 Issue of HR. Journal of Travel Research, vol. 39, 319-320.

HOSTELTUR (2016): Cataluña recauda 43,5 M $€$ por la tasa turística. Hosteltur. Disponible en: http://www.hosteltur.com/115924_cataluna-recauda-435-m-tasa-turistica.html

HUGHES, H. (1981): «A tourism tax the cases for and against», International Journal of Tourism Management, $\mathrm{n}^{\mathrm{o}} 2$ 2, 196-206.

IHALANAYAKE, R. (2007): Economics of tourism taxation: A study of tourism taxes in Australia. School of Applied Economics. Faculty of Business and Law Victoria University Melbourne, Australia. 381 pp. Disponible en: http://vuir.vu .edu.au/15608/1/ Ihalanayake_2007compressed.pdf 
INE (2016): Encuesta de ocupación de apartamentos turísticos. E.O.H. Encuesta de ocupación hotelera. E.O.A.T. Instituto Nacional de Estadística, Madrid.

JENSEN, T.C. y WANHILL, S. (2002): «Tourism's taxing times: value added tax in Europe and Denmark», Tourism Management, vol. 23 (1), 67-79.

KATO, A., KWAK, S. y MAK, J. (2010): «Using the Property Tax to Appropriate Gains from Tourism», Journal of Travel Research, vol. 50 (2). 144-153.

LASARTE, J. y ESEVERRI, E. (2001): Turismo y financiación autonómica y local: comentarios sobre la llamada "ecotasa” y otras alternativas de financiación. Granada: Comares.

LEY 58/2003, de 17 de diciembre, General Tributaria. Boletín Oficial del Estado, BOE de 18 de diciembre de 2003, núm.302, pp. 44987-45065.

LEY 5/2010, de 11 de Junio, de Autonomía Local de Andalucía. Boletín Oficial de la Junta de Andalucía, BOJA de 23 de junio de 2010, núm. 122, pp. 6-34.

LEY 5/2012, de 20 de marzo, de medidas fiscales, financieras y administrativas y de creación del Impuesto sobre las Estancias en Establecimientos Turísticos. Diario Oficial de la Generalidad de Cataluña, de 23 de marzo de 2012, núm. 6094, pp. 15369-15458. BOE núm. 83 de 06 de Abril de 2012,pp. 27915-28021

LEY 2/2016, de 30 de marzo, del impuesto sobre estancias turísticas en las Islas Baleares y de medidas de impulso del turismo sostenible. Boletín Oficial de las Islas Baleares, de 2 de abril de 2016, núm. 42,pp. 9110-9121.

MAK, J. y NISHIMURA, E. (1979): «The Economics of a Hotel Room Tax», Journal of Travel Research, $\mathrm{n}^{\circ} 17,2-6$.

MAK, J. (1988): «Taxing Hotel Room Rentals in the US», Journal of Travel Research, vol. 21, 10-15.

MERCADO, I. (2003): «Hacia un cambio de modelo turístico?: reflexiones a partir de la ecotasa», Revista de Fomento Social, vol. 58 (231), 469-500.

OMT (1998): Tourism taxation: Striking a fair deal. World Tourism Organization, Madrid (1998)

OMT (2017): Panorama OMT del turismo internacional. Edición 2016. España: OMT.

PAGE, S.J. (2009): Tourism management: managing for change. Butterworth Heinemann. Oxford.

PALMER, T. y RIERA, A. (2003): «Tourism and environmental taxes», Tourism Management, vol. 24, 665-674.

PALMER, T., RIERA, A. y ROSELLÓ, J. (2007): «Taxing tourism: The case of rental cars in Mallorca», Tourism Management, vol. 28 (1), 271-279.

PIGA, C. (2003): «Territorial planning and tourism development tax», Annals of Tourism Research, $\mathrm{n}^{\circ}$ vol. 30 (4), 886-905.

PONJAN, P. y THIRAWA, N. (2016): «Impacts of Thailand's tourism tax cut: A CGE analysis», Annals of Tourism Research, vol. 61, 45-62.

PRADOS, E. (2001): «Fiscalidad y turismo: el impuesto turístico», Quincena fiscal, $\mathrm{n}^{\circ}$ 4, 11-19.

PUIG, I. (2007): «Fiscalidad y sostenibilidad en los destinos turísticos, Estudios Turísticos, $\mathrm{n}^{\circ} 172-173,181-185$.

ROCA, J., PUIG, I. y HERCOWITZ, M. (2004): «La propuesta del impuesto turístico (Ecotasa) para Lanzarote», Revista de Estudios Regionales, $\mathrm{n}^{\circ}$ 70, 203-221. 
SAETA (2016): Datos básicos del turismo en Andalucía por provincias. Empresa Pública Turismo y Deporte de Andalucía (EPTA). Junta de Andalucía. Málaga.

SANTAMARTA, J. y CASTEJÓN, F. (2001): «La ecotasa de Baleares», Página Abierta, $\mathrm{n}^{\mathrm{o}} 116,12-17$.

SHEN, L. y TSUI, Y. (2009): «Taxing tourism: enhancing or reducing welfare?», Journal of Sustainable Tourism, vol. 17 (5), 627-635.

SIMANCAS, M. R. (2008): «El sistema de cobro de una tasa pública por la prestación de servicios turísticos en las áreas protegidas de Canarias», Cuadernos de Turismo, $n^{\circ} 21,201-238$.

SPENGER, J. y UYSAL, M. (1989): «Considerations in the hotel taxation process», Journal Hospitality Management, vol. 8 (4), 309-316. 
